\title{
Position sequences and a $q$-analogue for the modular hook length formula
}

\author{
Anthony Mendes \\ aamendes@calpoly.edu
}

\author{
Sam Lindbloom-Airey \\ slindblo@uw.edu
}

Department of Mathematics

California Polytechnic State University

San Luis Obispo, CA, 93407 U.S.A.

Submitted: Apr 23, 2019; Accepted: Oct 12, 2019; Published: Oct 25, 2019

(C) The authors. Released under the CC BY-ND license (International 4.0).

\begin{abstract}
We prove a $q$-analogue of the modular hook length formula using position sequences. These position sequences, which correspond to moving the beads in a mathematical abacus, provide a new combinatorial interpretation for the characters of the irreducible representations of the symmetric group.
\end{abstract}

Mathematics Subject Classifications: 05E05, 05E10, 20C30

\section{Introduction}

Let $n$ and $k$ be positive integers and let $\lambda$ and $\mu=\left(\mu_{1}, \mu_{2}, \ldots\right)$ be integer partitions of $n$. A rim hook of length $k$ is a sequence of $k$ connected cells in the (English) Young diagram for $\lambda$ that begins in a cell on the southeast boundary and travels up along the southeast edge such that its removal leaves the Young diagram of a smaller integer partition.

The sign of a rim hook $\rho$ is $(-1)^{(\text {the number of rows spanned by } \rho)-1}$. For example, below is a rim hook of length 6 with sign $(-1)^{3-1}=+1$ inside of the Young diagram of the integer partition $(7,6,4,3,1)$ :

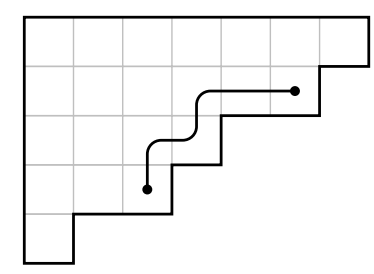

A rim hook tableau of shape $\lambda$ and content $\mu$ is a filling of the cells of the Young diagram of $\lambda$ with rim hooks of lengths $\mu_{1}, \mu_{2}, \ldots$ labeled with $1,2, \ldots$ such that the 
removal of the last $i$ rim hooks leaves the Young diagram of a smaller integer partition for all $i$. Let $R H T_{\mu}^{\lambda}$ be the set of all rim hook tableaux of shape $\lambda$ and content $\mu$.

The sign of a rim hook tableau $T$ is the product of all of the signs of the rim hooks in $T$. We let

$$
\chi_{\mu}^{\lambda}=\sum_{T \in R H T_{\mu}^{\lambda}} \operatorname{sign} T .
$$

For example, all rim hook tableaux of shape $(3,3,2,1)$ and content $(3,3,3)$ are:
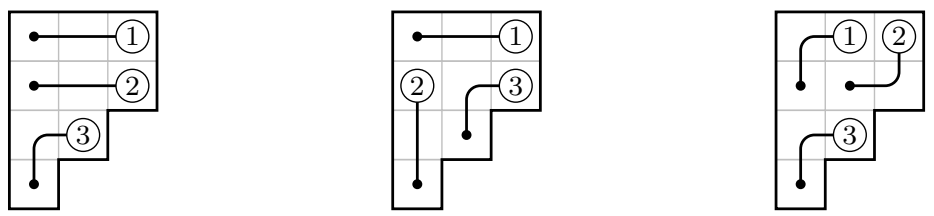

These three rim hook tableaux have sign -1 and so $\chi_{(3,3,3)}^{(3,3,2,1)}=-3$.

The numbers $\chi_{\mu}^{\lambda}$ are of significant interest because they give

1. the value of the irreducible character of $S_{n}$ indexed by $\lambda$ on $C_{\mu}$ where $C_{\mu}$ denotes the conjugacy class containing the permutations with cycle type $\mu$,

2. the coefficient of the Schur symmetric function $s_{\lambda}$ in the power symmetric function $p_{\mu}$, and

3. the coefficient of $\left|C_{\mu}\right| p_{\mu}$ in $n ! s_{\lambda}$.

As such, rim hook tableaux have been extensively studied and can be found in most treatments of the representation theory of the symmetric group $S_{n}$ and symmetric functions (see, for instance, $[11,14,9]$ ).

In the special case of $\mu=(1, \ldots, 1)$, rim hook tableaux of shape $\lambda$ and type $\mu$ are standard tableaux and the number $\chi_{(1, \ldots, 1)}^{\lambda}$ can be found using the hook length formula.

Theorem 1 (The hook length formula). If $\lambda$ is an integer partition of $n$, then

$$
\chi_{(1, \ldots, 1)}^{\lambda}=\frac{n !}{\prod_{c \in \lambda} h_{c}}
$$

where the notation $c \in \lambda$ means that $c$ is a cell in the Young diagram of $\lambda$ and the hook length $h_{c}$ is the length of the rim hook that begins in the same column as c and ends in the same row as $c$.

The hook length formula is a true crown jewel of enumerative combinatorics. Originally proved by Frame, Robinson, and Thrall [2], there are now a panoply of beautiful proofs (see final remark 10.3 and the references in [10]).

The hook length formula has been generalized in two different ways, the first of which involves the major index of a standard tableau. If $p=p_{1} \cdots p_{\ell}$ is any sequence of integers, the major index of $p$, denoted maj $p$, is equal to $\sum i$ where the sum runs over all indices 
$i$ such that $p_{i}>p_{i+1}$. Adapting this idea for standard tableaux, if $T \in R H T_{(1, \ldots, 1)}^{\lambda}$ is a standard tableau, then the integer $i$ is a descent in $T$ if rim hook $i$ appears in a row above that of rim hook $i+1$. The major index of $T$, denoted maj $T$, is equal to $\sum i$ where the sum runs over all descents $i$ in $T$.

For example, the descents in

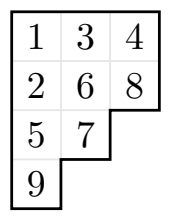

are $1,4,6$ and 8 , and so the major index is 19 .

The first generalization of the hook length formula is the $q$-hook length formula. It first appeared in [13] and was later proved using the elegant Hillman-Grassl algorithm [3].

Theorem 2 (The $\boldsymbol{q}$-hook length formula). If $\lambda=\left(\lambda_{1}, \lambda_{2}, \ldots\right)$ is an integer partition of $n$, then

$$
\sum_{T \in R H T_{(1, \ldots, 1)}^{\lambda}} q^{\operatorname{maj} T-\left(0 \lambda_{1}+1 \lambda_{2}+\cdots\right)}=\frac{[n]_{q} !}{\prod_{c \in \lambda}\left[h_{c}\right]_{q}}
$$

where $[n]_{q}=q^{0}+\cdots+q^{n-1}$ and $[n]_{q} !=[n]_{q} \cdots[1]_{q}$ are the usual $q$-analogues of $n$ and $n !$.

The second generalization of the hook length formula involves rim hook tableaux of shape $\lambda$ and content $(k, \ldots, k)$. These rim hook tableaux are useful in the modular representation theory of the symmetric group and can be used to generalize the RobinsonSchensted-Knuth (RSK) algorithm [15].

All rim hook tableaux of shape $\lambda$ with content $(k, \ldots, k)$ have the same sign (this is implied by (2.7.26) in [4]) and so $\left|\chi_{(k, \ldots, k)}^{\lambda}\right|$ is the number of rim hook tableaux of shape $\lambda$ and type $(k, \ldots, k)$. The value of this quantity is given by the modular hook length formula, first proved in [1]. The modular hook length formula is less well known than Theorem 1 but is beginning to receive the attention it deserves [16].

Theorem 3 (The modular hook length formula). If $\lambda$ is an integer partition of $n$ such that $\chi_{(k, \ldots, k)}^{\lambda} \neq 0$, then

$$
\left|\chi_{(k, \ldots, k)}^{\lambda}\right|=\frac{(n / k) !}{\prod h_{c} / k}
$$

where the product is over all cells $c \in \lambda$ with $h_{c}$ divisible by $k$.

The main result in this paper, Theorem 13, synthesizes the generalizations of the hook length formula in Theorems 2 and 3 to provide a $q$-analogue for the modular hook length formula. In order to prove Theorem 13 we introduce the concept of a position sequence. Position sequences are sequences of integers created from recording bead moves in a mathematical abacus. They provide a natural framework in which to understand rim hook tableaux, especially when interested in $q$-analogues. 
The outline of the paper is as follows. In section 2 we introduce position sequences, the main tool needed to prove the $q$-modular hook length formula. Interesting connections with the RSK algorithm are made, a position sequence version of Theorem 2 is given, and we find a $q$-analogue for the entire character table for the symmetric group in section 2 . Section 3 contains our proof of the $q$-modular hook length formula.

\section{Position sequences}

A $k$-abacus consists of $k$ runners, each of which is a sequence of beads and empty places. For example, this is a 3 -abacus with 7 beads:

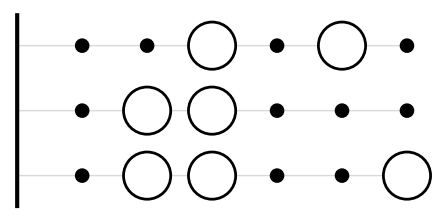

Starting in the bottom left corner, label the beads and the empty places in the abacus with the integers $1,2, \ldots$ by moving up each column, working left to right. For example, the above abacus is numbered

$$
\begin{array}{|llllll}
3 & 6 & 9 & 12 & 15 & 18 \\
2 & 5 & 8 & 11 & 14 & 17 \\
1 & 4 & 7 & 10 & 13 & 16
\end{array}
$$

and there are beads at positions 4, 5, 7, 8,9, 15 and 16 .

Each $k$-abacus $A$ represents an integer partition. Let $b_{1}, \ldots, b_{\ell}$ be the labels of the beads on a $k$-abacus and let empty $\left(b_{i}\right)$ be the number of empty places with a label smaller than $b_{i}$. Then the integer partition corresponding to $A$ is

$$
\lambda_{A}=\left(\operatorname{empty}\left(b_{\ell}\right), \ldots, \operatorname{empty}\left(b_{1}\right)\right) .
$$

For example, if $A$ is the 3 -abacus above, then $\lambda_{A}=(9,9,4,4,4,3,3)$.

Moving a bead in a $k$-abacus $A$ from position $i$ to an empty place in position $i-j$ removes a rim hook of length $j$ from $\lambda_{A}$. When $j=k$ this move sends a bead one step left on its runner. The sign of the removed rim hook is $(-1)^{b}$ where $b$ is the number of beads in positions between $i$ and $i-j$ (see Section 2.7 in [4]). These facts have been used to great effect in proving classic results from symmetric function theory using abaci $[6,7]$.

Therefore a rim hook tableau $T$ of shape $\lambda$ and content $\mu=\left(\mu_{1}, \ldots, \mu_{\ell}\right)$ can be interpreted as a sequence of bead moves in a $k$-abacus $A$ such that the $i^{\text {th }}$ bead move moves a bead in position $j$ for some $j$ to an empty place in position $j-\mu_{\ell+1-i}$ for $i=1, \ldots, \ell$. The beads in $A$ will be pushed as far as possible to the left after all of the moves. The sign of $T$ is the product of the signs of the bead moves.

We record such a sequence of bead moves with a position sequence. A position sequence $p=p_{1} \cdots p_{\ell}$ is the sequence of integers defined such that $p_{i}$ is the empty position filled by 
a bead on bead move $i$. If $A$ is a $k$-abacus with $\lambda_{A}=\lambda$, we let $P S_{\mu}^{\lambda}$ be the set of position sequences with bead moves of lengths given by $\mu$. It follows that $P S_{\mu}^{\lambda}$ and $R H T_{\mu}^{\lambda}$ have the same number of elements.

For example, one position sequence when $\lambda=(9,9,4,4,4,3,3)$ and $\mu=(3, \ldots, 3)$ is

$$
\begin{array}{lllllllllllll}
2 & 13 & 5 & 12 & 6 & 1 & 10 & 3 & 9 & 6 & 4 & 7 .
\end{array}
$$

Starting with the 3-abacus displayed above, this position sequence says to move the bead in position 5 into position 2, then move the bead in position 16 to position 13, then move the bead in position 8 into position 5 , and so on. The rim hook tableau of shape $\lambda$ and content $\mu$ for this position sequence is:

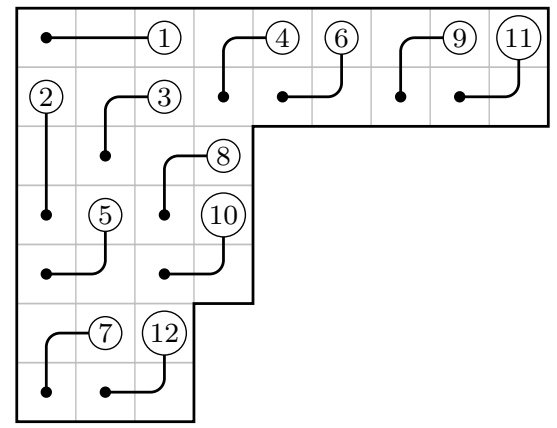

This rim hook tableau was created by finding $\lambda_{A}$ after each bead move and placing a rim hook in the removed cells.

The position sequence in (2) contains the subsequence $\begin{array}{lllll}2 & 6 & 3 & 9 & 6 \text {. This subsequence }\end{array}$ comes from moving beads within the $3^{\text {rd }}$ runner in the 3 -abacus (reading bottom to top), and so these numbers are congruent to 3 modulo 3 . Furthermore, the position sequence contains the subsequences $6 \quad 9$ and $3 \quad 6$ because in order for the rightmost bead on the top runner to move into positions 9 and 6 , the leftmost bead on the top runner must already have moved into positions 6 and 3. With this example as a guide we work towards characterizing position sequences in $P S_{(k, \ldots, k)}^{\lambda}$ by their subsequences.

Let $\lambda=\left(\lambda_{1}, \ldots, \lambda_{\ell}\right)$ be an integer partition. For each $j=1, \ldots, \ell$, define $I_{j}$ to be the sequence created by listing the integers in the interval $\left[j, \lambda_{\ell-j+1}+j-1\right]$ in decreasing order. The sequence $I_{j}$ gives the positions that the $j^{\text {th }}$ bead in the 1-abacus will occupy when moved in a position sequence in $P S_{(1, \ldots, 1)}^{\lambda}$. For example, the corresponding labeled 1-abacus for $\lambda=(4,4,3,1)$ is

$$
\mid \begin{array}{llllllll}
1 & 2 & 3 & 4 & 5 & 6 & 7 & -8
\end{array}
$$

and the sequences $I_{1}, \ldots, I_{4}$ are

$$
I_{1}=1, \quad I_{2}=4 \quad 3 \quad 2, \quad I_{3}=6 \quad 5 \quad 4 \quad 3, \quad \text { and } \quad I_{4}=7 \quad 6 \quad 5 \quad 4 .
$$

A shuffle of the sequences $I_{1}, \ldots, I_{\ell}$ is a sequence created by interleaving $I_{1}, \ldots, I_{\ell}$ such that each of $I_{1}, \ldots, I_{\ell}$ appears as a subsequence. 
Lemma 4. Let $\lambda=\left(\lambda_{1}, \ldots, \lambda_{\ell}\right)$ be an integer partition. Then $p$ is a position sequence in $P S_{(1, \ldots, 1)}^{\lambda}$ if and only if $p$ is a shuffle of $I_{1}, \ldots, I_{\ell}$ and each integer $m$ in $p$ that comes from $I_{j}$ appears after every $m-1$ in $p$ coming from $I_{1}, \ldots, I_{j-1}$ for all $j=1, \ldots, \ell$.

Proof. Suppose $p$ is a position sequence in $P S_{(1, \ldots, 1)}^{\lambda}$. Since $I_{j}$ gives the positions the $j^{\text {th }}$ bead moves into in a sequence of bead moves that correspond to a position sequence, each $I_{j}$ appears as a subsequence in $p$ with the order of the integers in $I_{j}$ preserved. Therefore $p$ is a shuffle of $I_{1}, \ldots, I_{\ell}$.

If a bead is moved into position $m$ in a sequence of bead moves, then each of the beads to its left must already have been moved into in position $m-1$ or smaller. Therefore each integer $m$ in $p$ that comes from $I_{j}$ must appear after every integer $m-1$ that appears in each of $I_{1}, \ldots, I_{j-1}$.

Now suppose $p$ is a shuffle of $I_{1}, \ldots, I_{\ell}$ satisfying the condition in the statement of the theorem. The subsequence $I_{j}$ in $p$ represents moving the $j^{\text {th }}$ bead from its starting position to its final position, and condition in the statement of the theorem guarantees that position $m$ will be empty at the time when bead $j$ is moved into position $m$. Therefore $p$ represents a sequence of bead moves and so $p \in P S_{(1, \ldots, 1)}^{\lambda}$.

It will be convenient to break the $k$-abacus into $k$ instances of 1 -abaci. Let $\lambda^{(i)}$ be the integer partition found by considering the $i^{\text {th }}$ runner reading bottom to top on the $k$-abacus as a 1 -abacus. For example, $\lambda^{(1)}=(3,1,1), \lambda^{(2)}=(1,1)$, and $\lambda^{(3)}=(3,2)$ for the abacus in (1).

Theorem 5. Let $\lambda$ be an integer partition such that $R H T_{(k, \ldots, k)}^{\lambda}$ is nonempty. Let $P S_{k}^{\lambda^{(i)}}$ be the set of position sequences $p \in P S_{(1, \ldots, 1)}^{\lambda^{(i)}}$ with each integer $j$ in $p$ replaced with $k j+i$. Then $p$ is a position sequence in $P S_{(k, \ldots, k)}^{\lambda}$ if and only if $p$ is a shuffle of $p^{(1)}, \ldots, p^{(k)}$ for some $p^{(1)} \in P S_{k}^{\lambda^{(1)}}, \ldots, p^{(k)} \in P S_{k}^{\lambda^{(k)}}$.

Proof. Suppose $p \in P S_{(k, \ldots, k)}^{\lambda}$ and let $p^{(i)}$ be the subsequence of $p$ consisting of the values in $p$ congruent to $i$ modulo $k$. Each bead move on the $k$-abacus moves a bead on a runner one position to the left on the same runner. Therefore bead moves on a single runner must satisfy the conditions in Lemma 4 , showing that $p^{(i)} \in P S_{k}^{\lambda^{(i)}}$ for $i=1, \ldots, \ell$. This shows that $p$ is a shuffle of $p^{(1)}, \ldots, p^{(k)}$ for some $p^{(1)} \in P S_{k}^{\lambda^{(1)}}, \ldots, p^{(k)} \in P S_{k}^{\lambda^{(k)}}$.

Now suppose $p$ is a shuffle of $p^{(1)}, \ldots, p^{(k)}$ for some $p^{(1)} \in P S_{k}^{\lambda^{(1)}}, \ldots, p^{(k)} \in P S_{k}^{\lambda^{(k)}}$. Since bead moves on different runners do not influence each other, it follows from 4 that $p$ corresponds to a sequence of bead moves on the $k$-abacus and so $p \in P S_{(k, \ldots, k)}^{\lambda}$.

An increasing run in a sequence of integers is a maximal weakly increasing consecutive subsequence.

Lemma 6. Let $A_{1}, \ldots, A_{\ell}$ be finite sequences of integers. We interpret each of $A_{1}, \ldots, A_{\ell}$ as having the same number $r$ of increasing runs by possibly padding the beginning of each of $A_{1}, \ldots, A_{\ell}$ with empty increasing runs. Define $\hat{p}$ to be the shuffle of $A_{1}, \ldots, A_{\ell}$ with 
$r$ increasing runs such that the $i^{\text {th }}$ increasing run in $\hat{p}$ contains the integers in the $i^{\text {th }}$ increasing runs in each of $A_{1}, \ldots, A_{\ell}$ sorted into increasing order for $i=1, \ldots, r$. Then

$$
\operatorname{maj} \hat{p}=\operatorname{maj}\left(A_{1}\right)+\cdots+\operatorname{maj}\left(A_{\ell}\right)
$$

and this $\hat{p}$ is the unique shuffle of $A_{1}, \ldots, A_{\ell}$ with the minimum possible major index.

As an example, consider

$$
A_{1}=1 \quad 2 \quad 4 \quad 3 \quad 6, \quad A_{2}=3 \quad 2 \quad 3 \quad 3, \quad \text { and } \quad A_{3}=1 \quad 5 \quad 5 .
$$

Here $A_{1}$ and $A_{2}$ have 2 nonempty increasing runs and $A_{3}$ has 1 nonempty increasing run, so we interpret $A_{3}$ as having 2 increasing runs where the first increasing run is empty. Then we see $\hat{p}=123412333556$ and maj $\hat{p}=4=3+1+0=\operatorname{maj} A_{1}+$ maj $A_{2}+$ maj $A_{3}$.

Proof. The assertion is trivially true when each of $A_{1}, \ldots, A_{\ell}$ is empty. We proceed by induction on $\left|A_{1}\right|+\cdots+\left|A_{\ell}\right|$.

Let $\widetilde{A_{j}}$ be $A_{j}$ with its final increasing run removed. The only descent in $A_{j}$ that does not appear in $\widetilde{A_{j}}$ is in position $\left|A_{j}\right|-\left|\widetilde{A_{j}}\right|$ and so

$$
\operatorname{maj} \widetilde{A_{j}}+\left|A_{j}\right|-\left|\widetilde{A_{j}}\right|=\operatorname{maj} A_{j}
$$

for $j=1, \ldots, \ell$.

Let $p$ be a shuffle of $A_{1}, \ldots, A_{\ell}$. There must be a descent in $p$ at position

$$
\left|A_{1}\right|+\cdots+\left|A_{\ell}\right|-\left|\widetilde{A_{1}}\right|-\cdots-\left|\widetilde{A_{\ell}}\right|
$$

or greater because this is the position where the maximum possible final increasing run in any shuffle of $A_{1}, \ldots, A_{\ell}$ begins (this maximum possible final increasing run is created by combining the final increasing runs in $A_{1}, \ldots, A_{\ell}$ into one increasing sequence). If we define $p^{\prime}$ to be $p$ with its final increasing run removed, then this implies

$$
\operatorname{maj} p \geqslant\left|A_{1}\right|+\cdots+\left|A_{\ell}\right|-\left|\widetilde{A_{1}}\right|-\cdots-\left|\widetilde{A_{\ell}}\right|+\operatorname{maj} p^{\prime}
$$

where equality is achieved only when the final descent in $p$ occurs in the position in (5).

Let $A_{1}^{\prime}, \ldots, A_{\ell}^{\prime}$ be $A_{1}, \ldots, A_{\ell}$ but with possibly some of their tails trimmed so that $p^{\prime}$ is a shuffle of $A_{1}^{\prime}, \ldots, A_{\ell}^{\prime}$. Then $\widetilde{A_{j}}$ is equal to $A_{j}^{\prime}$ but maybe with some final integers deleted. Therefore we have maj $A_{j}^{\prime} \geqslant$ maj $\widetilde{A_{j}}$ for each $j=1, \ldots, \ell$.

The induction hypothesis on $p^{\prime}$ gives that maj $p^{\prime} \geqslant \operatorname{maj} A_{1}^{\prime}+\cdots+$ maj $A_{\ell}^{\prime}$ with equality holding if and only if $p^{\prime}$ is the unique shuffle of $A_{1}^{\prime}, \ldots, A_{\ell}^{\prime}$ with the minimum possible major index as described in the statement of the Lemma.

Putting these observations together gives

$$
\begin{aligned}
\operatorname{maj} p & \geqslant\left|A_{1}\right|+\cdots+\left|A_{\ell}\right|-\left|\widetilde{A_{1}}\right|-\cdots-\left|\widetilde{A_{\ell}}\right|+\operatorname{maj} p^{\prime} \\
& \geqslant\left|A_{1}\right|+\cdots+\left|A_{\ell}\right|-\left|\widetilde{A_{1}}\right|-\cdots-\left|\widetilde{A_{\ell}}\right|+\operatorname{maj} A_{1}^{\prime}+\cdots+\operatorname{maj} A_{\ell}^{\prime} \\
& \geqslant\left|A_{1}\right|+\cdots+\left|A_{\ell}\right|-\left|\widetilde{A_{1}}\right|-\cdots-\left|\widetilde{A_{\ell}}\right|+\operatorname{maj} \widetilde{A_{1}}+\cdots+\operatorname{maj} \widetilde{A_{\ell}} \\
& =\operatorname{maj} A_{1}+\cdots+\operatorname{maj} A_{\ell}
\end{aligned}
$$


where the last line used (4).

Equality is achieved in this string of inequalities if and only if there is a descent exactly in the position in (5) (hence $A_{j}^{\prime}=\widetilde{A_{j}}$ for all $j$ ) and $p^{\prime}$ is the unique shuffle of $A_{1}^{\prime}, \ldots, A_{\ell}^{\prime}$ with the minimum possible major index. In other words, equality is uniquely achieved when the shuffle $p$ is the shuffle $\hat{p}$ as described in the statement of the Lemma.

Corollary 7. Let $\hat{p}$ be the position sequence in $P S_{(1, \ldots, 1)}^{\lambda}$ with the minimum major index. Then $\hat{p}$ corresponds to finding the rightmost bead $b$ in the 1-abacus that can be moved one position to the left, moving $b$ one position to the left, and then iterating until no more moves can be made.

Proof. Since $I_{j}$ has length $\lambda_{\ell-j+1}$, the sequences $I_{1}, \ldots, I_{\ell}$ weakly increase in length. Let $m$ be the minimum integer such that the sets $I_{m}, \ldots, I_{\ell}$ all have the same length. This means that $\lambda$ has $\ell-m+1$ copies of its largest part, and, on the 1-abacus, the sequence of beads and empty places ends with an empty place and then $\ell-m+1$ consecutive beads.

Let $z$ be the first integer in $I_{m}$. If $b$ is the rightmost bead in the 1-abacus that can be moved one position to the left, then the appearance of $z$ in a position sequence corresponds to moving $b$ one position to the left. It remains to be shown that $\hat{p}$ begins with $z$.

Since each of $I_{1}, \ldots, I_{m-1}$ has a length less than that of $I_{m}$, we begin creating $\hat{p}$ by padding each of $I_{1}, \ldots, I_{m-1}$ with empty increasing runs. Then $z$ appears first in $\hat{p}$ because $z$ is the minimum integer appearing in the first increasing runs of $I_{1}, \ldots, I_{\ell}$.

Theorem 8. If $\hat{p}$ is the element in $P S_{(k, \ldots, k)}^{\lambda}$ with the minimum major index, then

$$
\operatorname{maj} \hat{p}=\sum\left(\begin{array}{l}
x \\
2
\end{array}\right)
$$

where the sum runs over all parts $x$ in the integer partitions $\lambda^{(1)}, \ldots, \lambda^{(k)}$.

Proof. Suppose $\lambda^{(i)}=\left(\lambda_{1}^{(i)}, \ldots, \lambda_{\ell_{i}}^{(i)}\right)$ and let $p^{(i)}$ be an element of $P S_{k}^{\lambda^{(i)}}$. By Lemma 4, $p^{(i)}$ has $k I_{j}+i$ as subsequence for $j=1, \ldots, \ell_{i}$. This subsequence has length $\lambda_{\ell_{i}-j+1}^{(i)}$, so it has major index $1+2+\cdots+\left(\lambda_{\ell_{i}-j+1}^{(i)}-1\right)=\left(\begin{array}{c}\lambda_{\ell_{i}-j+1}^{(i)} \\ 2\end{array}\right)$. Using Lemma 6 on the sequences $k I_{1}+i, \ldots, k I_{\ell_{i}}+i$ gives that the minimum major index over all elements in $P S_{k}^{\lambda^{(i)}}$ is

$$
\left(\begin{array}{c}
\lambda_{1}^{(i)} \\
2
\end{array}\right)+\cdots+\left(\begin{array}{c}
\lambda_{\ell_{i}}^{(i)} \\
2
\end{array}\right) .
$$

The second condition in Lemma 4 says that $m$ must appear after a certain number of appearances of $m-1$. As can be seen using Corollary 7, this condition is preserved when using Lemma 6 . Therefore this minimum major index is actually achieved by an element in $P S_{k}^{\lambda^{(i)}}$.

By Theorem 5, each element in $P S_{(k, \ldots, k)}^{\lambda}$ is a shuffle of sequences $p^{(1)}, \ldots, p^{(k)}$ with $p^{(i)} \in P S_{k}^{\lambda^{(i)}}$. Taking each $p^{(i)}$ to be the element with major index given in (6), another application of Lemma 6 completes the proof. 
As an example of Theorem 8, consider the $k$-abacus in $(1)$. Since $\lambda^{(1)}=(3,1,1)$, $\lambda^{(2)}=(1,1)$, and $\lambda^{(3)}=(3,2)$, the minimum major index is $\left(\begin{array}{l}3 \\ 2\end{array}\right)+\left(\begin{array}{l}1 \\ 2\end{array}\right)+\cdots+\left(\begin{array}{l}3 \\ 2\end{array}\right)+\left(\begin{array}{l}2 \\ 2\end{array}\right)=7$. Lemma 6 and the proof of Theorem 8 tell us that the unique position sequence $\hat{p}$ that

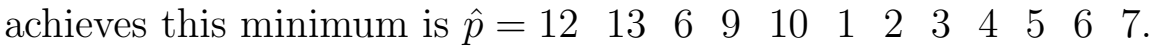

The RSK algorithm can be used to understand monotonic subsequences in words. Lemma 4 characterizes position sequences in terms of decreasing subsequences, and so it may not come as a surprise that there is a relationship between the RSK algorithm and position sequences.

Theorem 9. The RSK algorithm produces the same insertion tableau $P$ for every position sequence in $P S_{(1, \ldots, 1)}^{\lambda}$.

Proof. We will use Knuth equivalence, defined as follows. Let $A$ and $B$ be finite sequences with integer letters. An elementary Knuth operation on $A$ is one of these two operations or their inverses:

1. If $x z y$ appears consecutively in $A$ and $x \leqslant y<z$, then the order of these letters is changed to $z x y$ and the rest of $A$ is left unchanged.

2. If $y z x$ appears consecutively in $A$ and $x<y \leqslant z$, then the order of these letters is changed to $y x z$ and the rest of $A$ is left unchanged.

Then $A$ and $B$ are defined to be Knuth equivalent if $A$ can be transformed into $B$ by a sequence of elementary Knuth operations. This is relevant because $A$ and $B$ are Knuth equivalent if and only if the RSK algorithm produces the same $P$ tableau for $A$ and $B$ [5].

Let $\hat{p}$ be the position sequence in $P S_{(1, \ldots, 1)}^{\lambda}$ with the minimum major index and let $p$ be any other position sequence in $P S_{(1, \ldots, 1)}^{\lambda}$. We will prove the theorem by showing that $p$ and $\hat{p}$ are Knuth equivalent.

The theorem is clearly true when $|\lambda|$ is 0,1 or 2 because in these cases there is at most one position sequence in $P S_{(1, \ldots, 1)}^{\lambda}$. We will prove the theorem true when the length of $p$ is larger than 2 by induction on $|\lambda|$.

Let $z$ be the first integer in $\hat{p}$. If $p$ also begins with $z$, then we are done by induction on the remaining portion of $p$. If not, we will show that $z$ can be moved into the first position of $p$ using a sequence of elementary Knuth operations at which point the theorem again follows by induction on the remaining portion of $p$. For this it is enough to show that the leftmost appearance of $z$ in $p$ can be moved one position to the left in $p$ by a sequence of elementary Knuth operations.

Since $\hat{p}$ begins with $z$, position $z$ on the 1 -abacus is initially empty and remains empty when performing moves in the order given by $p$ until the move corresponding to the leftmost $z$. Furthermore, Corollary 7 implies that all integers larger than $z$ in $p$ appear to the right of the leftmost $z$. Therefore, if we define $x$ to be the integer immediately to the left of the leftmost $z$ in $p$, then $x+1<z$.

Let $p^{\prime}$ be the sequence of integers appearing to the right of the leftmost $z$ in $p$. Then $p^{\prime}$ is a position sequence in $P S_{(1, \ldots, 1)}^{\widetilde{\lambda}}$ for some integer partition $\widetilde{\lambda}$ such that $\widetilde{\lambda} \subset \lambda$. Let $p^{\prime \prime}$ be a position sequence in $P S_{(1, \ldots, 1)}^{\widetilde{\lambda}}$ that begins with an integer $y$ that satisfies $x \leqslant y<z$. 
Such a $p^{\prime \prime}$ exists because the integer $x+1$ appears to the right of the leftmost $z$ in $p$ (because the bead moves corresponding to the $x z$ in $p$ leave position $x+1$ empty and that empty position must eventually be filled with a bead). Thus one possible bead move that would correspond to an acceptable value of $y$ can be moving the leftmost possible bead to the right of $x$ first.

By induction, RSK produces the same insertion tableau $P$ for every position sequence in $P S_{(1, \ldots, 1)}^{\tilde{\lambda}}$ and therefore all position sequences in $P S_{(1, \ldots, 1)}^{\widetilde{\lambda}}$ are Knuth equivalent. In particular, there is a sequence of elementary Knuth operations that turns $p^{\prime}$ into $p^{\prime \prime}$. Applying these same elementary Knuth operations to $p$ yields a position sequence $p^{\prime \prime \prime} \in$ $P S_{(1, \ldots, 1)}^{\lambda}$ such that $p$ and $p^{\prime \prime \prime}$ are the same up until the leftmost $z$ and such that the tail end of $p^{\prime \prime \prime}$ is $p^{\prime \prime}$.

The sequence $p^{\prime \prime \prime}$ now contains the consecutive sequence $x z y$ with $x<y<z$. Using the first elementary Knuth operation, turn this into $z x y$. This still gives a valid position sequence because $z x y$ corresponds to moving a bead into position $z$ and then moving a bead into position $x$ instead of vice versa. We have now successfully moved the leftmost $z$ one position to the left using a sequence of Knuth operations, as needed.

For example, the insertion tableau $P$ found when applying the RSK algorithm to any $p \in P S_{(1, \ldots, 1)}^{\lambda}$ when $\lambda=(4,4,3,1)$ is

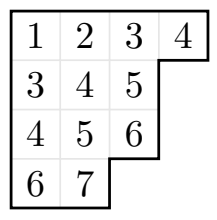

The reading word for a tableau is the word found by reading the rows left to right, bottom to top. The reading word for the above tableau is

$$
\begin{array}{llllllllllll}
6 & 7 & 4 & 5 & 6 & 3 & 4 & 5 & 1 & 2 & 3 & 4
\end{array}
$$

This word is also the position sequence $\hat{p} \in P S_{(1, \ldots, 1)}^{\lambda}$ with the minimum major index.

Theorem 10. Let $\lambda$ be an integer partition and let $P$ be the insertion tableau found when applying the $R S K$ algorithm to any position sequence in $P S_{(1, \ldots, 1)}^{\lambda}$. Then the shape of $P$ is the conjugate partition $\lambda^{\prime}$ and the reading word for $P$ is the position sequence $\hat{p} \in P S_{(1, \ldots, 1)}^{\lambda}$ with the minimum major index.

Proof. The position sequence $\hat{p}$ with the minimum major index is the reading word for some standard tableau, say $\hat{P}$. The construction of $\hat{p}$ given in Lemma 6 implies that $\hat{P}$ has shape $\lambda^{\prime}$ because the parts of $\lambda^{\prime}$ give the lengths of the increasing runs in $\hat{p}$.

Lemma 3.4.5 in [11] says that the insertion tableau $P$ found when applying the RSK algorithm to $\hat{p}$ is also $\hat{P}$, and Theorem 9 says that all position sequences in $P S^{\lambda}$ have the same insertion tableau $P$. 
Theorem 11 (The position sequence version of the $\boldsymbol{q}$-hook length formula). If $\lambda=$ $\left(\lambda_{1}, \ldots, \lambda_{\ell}\right)$ is an integer partition of $n$ and $\hat{p}$ is the position sequence in $P S_{(1, \ldots, 1)}^{\lambda}$ with the minimum major index, then

$$
\sum_{p \in P S_{(1, \ldots, 1)}^{\lambda}} q^{\operatorname{maj} p-\operatorname{maj} \hat{p}}=\frac{[n]_{q} !}{\prod_{c \in \lambda}\left[h_{c}\right]_{q}} .
$$

Proof. There are the same number of position sequences in $P S_{(1, \ldots, 1)}^{\lambda}$ as there are rim hook tableaux of shape $\lambda$ and type $(1, \ldots, 1)$, which is also the number of rim hook tableaux of shape $\lambda^{\prime}$ and type $(1, \ldots, 1)$. If $P$ is the tableau of shape $\lambda^{\prime}$ with reading word $\hat{p}$ as in Theorem 10, then

$$
\left\{(P, Q): Q \text { is a rim hook tableau of shape } \lambda^{\prime} \text { and type }(1, \ldots, 1)\right\}
$$

has the same size as $P S_{(1, \ldots, 1)}^{\lambda}$. Since RSK is a bijection, applying RSK to $P S_{(1, \ldots, 1)}^{\lambda}$ produces every element in (7) exactly once.

If $p$ is any word and RSK sends $p$ to $(P, Q)$, then maj $p=\operatorname{maj} Q$ where maj $p$ is the major index for sequences and maj $Q$ is the major index for standard tableaux (see, for instance, [7]). This, combined with Theorem 8 , gives

$$
\sum_{p \in P S_{(1, \ldots, 1)}^{\lambda}} q^{\text {maj } p-\operatorname{maj} \hat{p}}=\sum_{Q \in R H T_{(1, \ldots, 1)}^{\lambda^{\prime}}} q^{\operatorname{maj} Q-\left(\begin{array}{c}
\lambda_{1} \\
2
\end{array}\right)-\cdots-\left(\begin{array}{c}
\lambda_{\ell} \\
2
\end{array}\right) .}
$$

Let $\lambda^{\prime}=\left(\lambda_{1}^{\prime}, \ldots, \lambda_{r}^{\prime}\right)$ be the conjugate partition to $\lambda$. Consider the tableau of shape $\lambda^{\prime}$ with $i-1$ occupying every entry in row $i$, like this example when $\lambda=(4,4,3,1)$ :

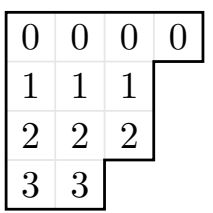

The sum of column $j$ is $\left(\begin{array}{c}\lambda_{j} \\ 2\end{array}\right)$. Summing the entries in this tableau column by column and then row by row, we find $\left(\begin{array}{c}\lambda_{1} \\ 2\end{array}\right)+\left(\begin{array}{c}\lambda_{2} \\ 2\end{array}\right)+\cdots=0 \lambda_{1}^{\prime}+1 \lambda_{2}^{\prime}+\cdots$. Using this in (8) and then applying Theorem 2 gives

$$
\sum_{Q \in R H T_{(1, \ldots, 1)}^{\lambda^{\prime}}} q^{\operatorname{maj} Q-\left(0 \lambda_{1}^{\prime}+1 \lambda_{2}^{\prime}+\cdots\right)}=\frac{[n]_{q} !}{\prod_{c \in \lambda^{\prime}}\left[h_{c}\right]_{q}}=\frac{[n]_{q} !}{\prod_{c \in \lambda}\left[h_{c}\right]_{q}},
$$

as needed.

Although this paper is most concerned with the situation where $\mu=(k, \ldots, k)$, we can use position sequences to give a $q$-analogue for $\chi_{\mu}^{\lambda}$ when $\mu \neq(k, \ldots, k)$. The sign of a bead move from position $i$ to position $i-j$ is $(-1)^{b}$ where $b$ is the number of beads in 
positions between $i$ and $i-j$. We define $\operatorname{sign} p$ to be the product of the signs of all of the bead moves given by the position sequence $p$. A $q$-analogue for $\chi_{\mu}^{\lambda}$ can be defined as

$$
\chi_{\mu, q}^{\lambda}=\sum_{p \in P S_{\mu}^{\lambda}}(\operatorname{sign} p) q^{\operatorname{maj} p} .
$$

For example, consider $\lambda=\left(3,1^{2}\right)$. The labeled 1 -abacus for $\lambda$ is

$$
\mid \begin{array}{llllll}
1 & 2 & 3 & 4 & 5 & 6
\end{array} \text {. }
$$

We have $\chi_{\left(2,1^{3}\right), q}^{\left(3,1^{2}\right)}=q+q^{2}-q^{4}-q^{5}$, found by moving beads of distances $1,1,1$ and 2 . The relevant position sequences are below:

\begin{tabular}{cccccc}
\hline Position & Sequence & Sign & Major Index \\
\hline 5 & 1 & 2 & 3 & +1 & 1 \\
1 & 5 & 2 & 3 & +1 & 2 \\
1 & 2 & 5 & 3 & +1 & 3 \\
5 & 4 & 1 & 2 & -1 & 3 \\
5 & 1 & 4 & 2 & -1 & 4 \\
1 & 5 & 4 & 2 & -1 & 5 \\
\hline
\end{tabular}

Doing this type of calculation as $\lambda$ and $\mu$ range over all integer partitions of 5 gives a $q$-analogue for the character table for $S_{5}$ :

\begin{tabular}{c|ccccccc}
\hline & $C_{\left(1^{5}\right)}$ & $C_{\left(2,1^{3}\right)}$ & $C_{\left(2^{2}, 1\right)}$ & $C_{\left(3,1^{2}\right)}$ & $C_{(3,2)}$ & $C_{(4,1)}$ & $C_{(5)}$ \\
\hline$\chi^{(5)}$ & $q^{10}$ & $q^{6}$ & $q^{3}$ & $q^{3}$ & $q$ & $q$ & 1 \\
$\chi^{(4,1)}$ & $q^{6}[4]_{q}$ & $q^{3}+q^{4}+q^{5}-q^{6}$ & $q^{2}-q^{3}$ & $q+q^{2}-q^{3}$ & $-q$ & $1-q$ & -1 \\
$\chi^{(3,2)}$ & $q^{4}[5]_{q}$ & $q^{2}+q^{3}-q^{5}$ & $q-q^{2}+q^{3}$ & $q-q^{2}-q^{3}$ & 1 & $-q$ & 0 \\
$\chi^{\left(3,1^{2}\right)}$ & $q^{3} \frac{[4]_{q}[3]_{q}}{[2]_{q}}$ & $q+q^{2}-q^{4}-q^{5}$ & $-q-q^{2}$ & $1-q^{2}+q^{3}$ & $q-1$ & $q-1$ & 1 \\
$\chi^{\left(2^{2}, 1\right)}$ & $q^{2}[5]_{q}$ & $q-q^{3}-q^{4}$ & $1-q+q^{2}$ & $-q$ & $-q$ & $q$ & 0 \\
$\chi^{\left(2,1^{3}\right)}$ & $q[4]_{q}$ & $1-q-q^{2}-q^{3}$ & $q-1$ & $-1+q+q^{2}$ & 1 & $1-q$ & -1 \\
$\chi^{\left(1^{5}\right)}$ & 1 & -1 & 1 & 1 & -1 & -1 & 1 \\
\hline
\end{tabular}

The $\chi^{\lambda}$ row and $C_{\mu}$ column entry is $\chi_{\mu, q}^{\lambda}$. The first column was found using Theorem 11.

If $\nu$ is a rearrangement of the parts in $\mu$, then $\chi_{\mu}^{\lambda}=\chi_{\nu}^{\lambda}$ (see [15, 8]). Unfortunately, the $q$-analogue $\chi_{\mu, q}^{\lambda}$ does not enjoy the same property. For example, we have $\chi_{(1,1,1,2), q}^{\left(3,1^{2}\right)}=q-q^{5}$ because the position sequence $4 \quad 1 \quad 2 \quad 3$ with sign +1 has major index 1 and the position sequence $154 \quad 4 \quad 3$ with sign -1 has major index 5 . 


\section{The $q$-modular hook length formula}

After this next Lemma we will be ready to prove our main result in Theorem 13.

Lemma 12. If $\lambda$ is an integer partition and $P S_{k}^{\lambda^{(i)}}$ is as in Theorem 5, then

$$
\frac{\sum_{p \in P S_{(k, \ldots, k)}^{\lambda}} q^{\text {maj } p}}{(1-q) \cdots\left(1-q^{|\lambda| / k}\right)}=\prod_{i=1}^{k} \frac{\sum_{p^{(i)} \in P S_{k}^{\lambda}}(1-q) \cdots\left(1-q^{\left|\lambda^{(i)}\right|}\right)}{\left(1-{ }^{\operatorname{maj} p^{(i)}}\right.} .
$$

Proof. The $1 /\left((1-q) \cdots\left(1-q^{|\lambda| / k}\right)\right)$ term on the left side of $(9)$ is the generating function for integer partitions with no part larger than $|\lambda| / k$. Conjugating such an integer partition gives an integer partition that has exactly $|\lambda| / k$ parts with parts of size 0 allowed. The length of $p \in P S_{(k, \ldots, k)}^{\lambda}$ is $|\lambda| / k$ and so the left side of (9) is equal to

$$
\sum q^{\operatorname{maj} p+|\pi|}
$$

where the sum runs over all possible $p \in P S_{(k, \ldots, k)}^{\lambda}$ and all possible integer partitions $\pi$ with parts of size 0 allowed such that the lengths of $\pi$ and $p$ are the same. Similarly, the right side of (9) is equal to

$$
\sum q^{\operatorname{maj} p^{(1)}+\cdots+\operatorname{maj} p^{(k)}+\left|\pi^{(1)}\right|+\cdots+\left|\pi^{(k)}\right|}
$$

where the sum runs over all possible $p^{(i)} \in P S_{k}^{\lambda^{(i)}}$ and all possible integer partitions $\pi^{(1)}, \ldots, \pi^{(k)}$ with parts of size 0 allowed such that the lengths of $\pi^{(i)}$ and $p^{(i)}$ are the same for each $i$.

We will prove the lemma by exhibiting a bijection $\varphi$ which sends pairs of the form $(p, \pi)$ where $p$ is a position sequence in $P S_{(k, \ldots, k)}^{\lambda}$ and $\pi$ is an integer partition with 0 parts allowed such that $p$ and $\pi$ have the same length to tuples of the form $\left(p^{(1)}, \pi^{(1)}, \ldots, p^{(k)}, \pi^{(k)}\right)$ where $p^{(i)} \in P S_{k}^{\lambda^{(i)}}$ and $\pi^{(i)}$ have the same length for each $i$. The bijection $\varphi$ will have the weight preserving property that

$$
\operatorname{maj} p+|\pi|=\operatorname{maj} p^{(1)}+\cdots+\operatorname{maj} p^{(k)}+\left|\pi^{(1)}\right|+\cdots+\left|\pi^{(k)}\right| .
$$

Let $p=p_{1} p_{2} \ldots$ be a position sequence in $P S_{(k, \ldots, k)}^{\lambda}$ and $\pi=\left(\pi_{1}, \pi_{2}, \ldots\right)$ be an integer partition with 0 parts allowed such that $p$ and $\pi$ have the same length. Let $c(i, j)$ be the position of the $j^{\text {th }}$ integer in $p$ that is congruent to $i$ modulo $k$. Define $p^{(i)}=p_{1}^{(i)} p_{2}^{(i)} \ldots$ where $p_{j}^{(i)}=p_{c(i, j)}$ and define $\pi^{(i)}=\left(\pi_{1}^{(i)}, \pi_{2}^{(i)}, \ldots\right)$ where

$$
\begin{aligned}
\pi_{j}^{(i)}=\pi_{c(i, j)} & + \text { (the number of descents in } p \text { at position } c(i, j) \text { or greater }) \\
& -\left(\text { the number of descents in } p^{(i)} \text { at position } j \text { or greater }\right)
\end{aligned}
$$

The bijection $\varphi$ is defined to send $(p, \pi)$ to $\left(p^{(1)}, \pi^{(1)}, \ldots, p^{(k)}, \pi^{(k)}\right)$.

For example, suppose $\lambda=(9,9,4,4,4,3,3), k=3$, 


$$
\begin{array}{lcccccccccccc}
p= & 2 & 13 & 5 & 12 & 6 & 1 & 10 & 3 & 9 & 6 & 4 & 7 \text { and } \\
\pi= & (2, & 1, & 1, & 1, & 1, & 1, & 1, & 1, & 1, & 0, & 0, & 0) .
\end{array}
$$

Then it can be found that

$$
\begin{aligned}
& p^{(1)}=13 \quad 1 \quad 10 \quad 4 \quad 7, \quad p^{(2)}=2 \quad 5, \quad p^{(3)}=12 \quad 6 \quad 3 \quad 9 \quad 6, \\
& \pi^{(1)}=(5, \quad 3, \quad 3, \quad 0, \quad 0), \quad \pi^{(2)}=(8, \quad 6), \quad \pi^{(3)}=(3, \quad 3, \quad 2, \quad 2, \quad 1),
\end{aligned}
$$

and we see that $\operatorname{maj} p+|\pi|=47=\operatorname{maj} p^{(1)}+\operatorname{maj} p^{(2)}+\operatorname{maj} p^{(3)}+\left|\pi^{(1)}\right|+\left|\pi^{(2)}\right|+\left|\pi^{(3)}\right|$.

The sequences $p^{(1)}, \ldots, p^{(k)}$ are indeed elements in $P S_{k}^{\lambda^{(i)}}$ because of Theorem 5 .

Suppose $p^{(i)}$ has a descent in position $m \geqslant j$. Since $p$ is a shuffle of $p^{(1)}, \ldots, p^{(k)}$, the integer $p_{m}^{(i)}$ appears to the left of $p_{m+1}^{(i)}$ in $p$, and so there must be at least one descent between $p_{m}^{(i)}$ and $p_{m+1}^{(i)}$ in $p$. Thus every descent in $p^{(i)}$ in position $j$ or greater has at least one corresponding descent in $p$ in position $c(i, j)$ or greater. Therefore

(the number of descents in $p$ at position $c(i, j)$ or greater)

is at least as large as

(the number of descents in $p^{(i)}$ at position $j$ or greater)

and the difference of these quantities weakly decreases as $j$ increases. We can now conclude that $\pi^{(1)}, \ldots, \pi^{(k)}$ are indeed integer partitions.

The function $\varphi$ is a bijection because we can describe its inverse. Suppose we are given the tuple

$$
\left(p^{(1)}, \pi^{(1)}, \ldots, p^{(k)}, \pi^{(k)}\right)
$$

where $p^{(i)} \in P S_{k}^{(i)}$ and $\pi^{(i)}$ have the same length for each $i$. Define $\widehat{\pi}^{(i)}=\left(\widehat{\pi}_{1}^{(i)}, \widehat{\pi}_{2}^{(i)}, \ldots\right)$ to be the integer partition such that

$$
\widehat{\pi}_{j}^{(i)}=\pi_{j}^{(i)}+\left(\text { the number of descents in } p^{(i)} \text { at position } j \text { or greater }\right) .
$$

This definition of $\widehat{\pi}^{(i)}$ increments each of the $j$ parts $\pi_{1}^{(i)}, \ldots, \pi_{j}^{(i)}$ by 1 if there is a descent in $p^{(i)}$ at position $j$, and so $\left|\widehat{\pi}^{(i)}\right|=\operatorname{maj} p^{(i)}+\left|\pi^{(i)}\right|$. Furthermore, this definition implies that $p_{j}^{(i)}<p_{j+1}^{(i)}$ for every value of $j$ that satisfies $\widehat{\pi}_{j}^{(i)}=\widehat{\pi}_{j+1}^{(i)}$.

Define $\widehat{\pi}=\left(\widehat{\pi}_{1}, \widehat{\pi}_{2}, \ldots\right)$ to be the integer partition found by sorting the parts of $\widehat{\pi}^{(1)}, \ldots, \widehat{\pi}^{(k)}$ into weakly decreasing order. Define $p=p_{1} p_{2} \cdots$ to be the shuffle of $p^{(1)}, \ldots, p^{(k)}$ such that $p_{j}^{(i)}$ appears in the same position in $p$ as $\widehat{\pi}_{j}^{(i)}$ appears in $\widehat{\pi}$ and such that $p_{j}<p_{j+1}$ for every value of $j$ that satisfies $\widehat{\pi}_{j}=\widehat{\pi}_{j+1}$.

If $p_{j}>p_{j+1}$, then $\widehat{\pi}_{j}>\widehat{\pi}_{j+1}$, and so

$$
\widehat{\pi}_{j} \geqslant \text { (the number of descents in } p \text { at position } j \text { or greater) }
$$

for all $j$. Define the integer partition $\pi=\left(\pi_{1}, \pi_{2}, \ldots\right)$ such that

$$
\pi_{j}=\widehat{\pi}_{j}-\text { (the number of descents in } p \text { at position } j \text { or greater). }
$$


The function $\varphi$ sends the pair $(p, \pi)$ to the tuple in (11) because this pair can easily be used to find the above $\widehat{\pi}$ and $p^{(i)}$, which in turn can be used to show that the above $\pi_{j}^{(i)}$ matches that given in (10). Therefore $\varphi$ is a bijection.

Since $\widehat{\pi}$ is decremented by 1 for each position $j$ of a descent in $p$, we have $|\pi|=$ $|\widehat{\pi}|-\operatorname{maj} p$ and so

$$
\begin{aligned}
\operatorname{maj} p+|\pi| & =|\widehat{\pi}| \\
& =\left|\widehat{\pi}^{(1)}\right|+\cdots\left|\widehat{\pi}^{(k)}\right| \\
& =\operatorname{maj} p^{(1)}+\cdots+\operatorname{maj} p^{(k)}+\left|\pi^{(1)}\right|+\cdots+\left|\pi^{(k)}\right| .
\end{aligned}
$$

The bijection $\varphi$ is weight preserving, as needed.

Theorem 13 (The $\boldsymbol{q}$-modular hook length formula). If $\lambda$ is an integer partition of $n$ such that $P S_{(k, \ldots, k)}^{\lambda}$ is nonempty and $\hat{p}$ is the position sequence in $P S_{(k, \ldots, k)}^{\lambda}$ with the minimum major index, then

$$
\sum_{p \in P S_{(k, \ldots, k)}^{\lambda}} q^{\text {maj } p-\operatorname{maj} \hat{p}}=\frac{[n / k]_{q} !}{\prod\left[h_{c} / k\right]_{q}}
$$

where the product is over all cells $c \in \lambda$ with $h_{c}$ divisible by $k$.

Proof. After dividing both sides of Theorem 11 by $(1-q) \cdots\left(1-q^{\left|\lambda^{(i)}\right|}\right)$ and moving terms around, we have

$$
\frac{\sum_{p^{(i)} \in P S_{(1, \ldots, 1)}^{\lambda^{(i)}}} q^{\operatorname{maj} p^{(i)}}}{(1-q) \cdots\left(1-q^{\left|\lambda^{(i)}\right|}\right)}=q^{\operatorname{maj} \hat{p}^{(i)}} \prod_{c \in \lambda^{(i)}} \frac{1}{1-q^{h_{c}}} .
$$

Since the major index of a position sequence $p^{(i)} \in P S_{k}^{\lambda^{(i)}}$ is unchanged if each integer $j \in p^{(i)}$ is replaced with the integer $k j+i$, we can set $P S_{(1, \ldots, 1)}^{\lambda}$ in the above equation to $P S_{(1, \ldots, 1)}^{\lambda^{(i)}}$. Multiply the expressions together when each of $P S_{k}^{\lambda^{(1)}}, \ldots, P S_{k}^{\lambda^{(k)}}$ replaces $P S_{(1, \ldots, 1)}^{\lambda}$ in the above equation and then use Lemma 12 to arrive at

$$
\frac{\sum_{p \in P S_{(k, \ldots, k)}^{\lambda}} q^{\text {maj } p}}{(1-q) \cdots\left(1-q^{n / k}\right)}=\prod_{i=1}^{k} q^{\text {maj } \hat{p}^{(i)}} \prod_{c^{(i)} \in \lambda^{(i)}} \frac{1}{1-q^{h} c^{(i)}}
$$

where $\hat{p}^{(i)}$ is the position sequence in $P S_{k}^{\lambda^{(i)}}$ with the minimum major index.

Each cell $c \in \lambda$ corresponds to a pair $(e, b)$ such that $e$ is an empty position on the $k$-abacus, $b$ is a position of a bead on the $k$-abacus, and $e<b$. The hook length of $c$ is $b-e$. This is divisible by $k$ if and only if both positions $e$ and $b$ appear on the same runner of the $k$-abacus. Thus each cell $c \in \lambda$ with hook length $k j$ corresponds to a cell $c^{(i)} \in \lambda^{(i)}$ with hook length $j$. Therefore the right side of (12) is equal to

$$
q^{\operatorname{maj} \hat{p}^{(1)}+\cdots+\operatorname{maj} \hat{p}^{(k)}} \prod \frac{1}{1-q^{h_{c} / k}}
$$


where the product is over all cells $c \in \lambda$ with $h_{c}$ divisible by $k$.

There is a sequence of $n / k$ bead moves of length $k$ in the $k$-abacus that leave the empty partition (pushing all beads flush to the left) because $P S_{(k, \ldots, k)}^{\lambda}$ is nonempty, and so there are exactly $n / k$ such pairs $(e, b)$ where $e$ and $b$ are on the same runner. Any pairs $(e, b)$ where $e$ and $b$ are not on the same runner do not correspond to cells $c \in \lambda$ with $h_{c}$ divisible by $k$, and therefore there are exactly $n / k$ cells $c \in \lambda$ with $h_{c}$ divisible by $k$.

Theorem 8 implies maj $\hat{p}=\operatorname{maj} \hat{p}^{(1)}+\cdots+\operatorname{maj} \hat{p}^{(k)}$, and so we have now shown that

$$
\frac{\sum_{p \in P S_{(k, \ldots, k)}^{\lambda}} q^{\operatorname{maj} p}}{(1-q) \cdots\left(1-q^{n / k}\right)}=q^{\operatorname{maj} \hat{p}} \prod \frac{1}{1-q^{h_{c} / k}} .
$$

where the product is over all cells $c \in \lambda$ with $h_{c}$ divisible by $k$. The result follows after multiplying by $(1-q) \cdots\left(1-q^{n / k}\right)$ and simplifying.

\section{Acknowledgments}

The authors extend a warm and hearty thank you to the anonymous referee who provided an outstanding report with detailed errata and improvements. The mathematics community is well served by such a wonderful referee.

\section{References}

[1] S. Fomin and N. Lulov. On the number of rim hook tableaux. Zap. Nauchn. Sem. S.-Peterburg. Otdel. Mat. Inst. Steklov. (POMI), 223(Teor. Predstav. Din. Sistemy, Kombin. i Algoritm. Metody. I):219-226, 340, 1995.

[2] J. S. Frame, G. de B. Robinson, and R. M. Thrall. The hook graphs of the symmetric groups. Canadian J. Math., 6:316-324, 1954.

[3] A. P. Hillman and R. M. Grassl. Reverse plane partitions and tableau hook numbers. J. Combinatorial Theory Ser. A, 21(2):216-221, 1976.

[4] G. James and A. Kerber. The representation theory of the symmetric group, volume 16 of Encyclopedia of Mathematics and its Applications. Addison-Wesley Publishing Co., Reading, Mass., 1981.

[5] D. E. Knuth. Permutations, matrices, and generalized Young tableaux. Pacific J. Math., 34:709-727, 1970.

[6] N. A. Loehr. Abacus proofs of Schur function identities. SIAM J. Discrete Math., 24(4):1356-1370, 2010.

[7] N. A. Loehr. Bijective combinatorics. Discrete Mathematics and its Applications (Boca Raton). CRC Press, Boca Raton, FL, 2011.

[8] A. Mendes. The combinatorics of rim hook tableaux. Australas. J. Combin., 73:132$148,2019$. 
[9] A. Mendes and J. Remmel. Counting with symmetric functions, volume 43 of Developments in Mathematics. Springer, Cham, 2015.

[10] A. H. Morales, I. Pak, and G. Panova. Hook formulas for skew shapes I. $q$-analogues and bijections. J. Combin. Theory Ser. A, 154:350-405, 2018.

[11] B. E. Sagan. The symmetric group: representations, combinatorial algorithms, and symmetric functions., volume 203 of Graduate Texts in Mathematics. SpringerVerlag, New York, second edition, 2001.

[12] C. Schensted. Longest increasing and decreasing subsequences. Canad. J. Math., 13:179-191, 1961.

[13] R. P. Stanley. Theory and application of plane partitions. I, II. Studies in Appl. Math., 50:167-188; ibid. 50, 259-279, 1971.

[14] R. P. Stanley. Enumerative combinatorics. Vol. 2, volume 62 of Cambridge Studies in Advanced Mathematics. Cambridge University Press, Cambridge, 1999.

[15] D. W. Stanton and D. E. White. A Schensted algorithm for rim hook tableaux. J. Combin. Theory Ser. A, 40(2):211-247, 1985.

[16] J. P. Swanson. Standard tableaux and modular major index. Sém. Lothar. Combin., 78B:Art. 50, 9, 2017. 